\title{
Escala FDA para la medición de los factores del desarrollo adolescente y su predicción en el bienestar psicológico \\ FDA scale for the measurement of adolescent development factors and their prediction in psychological well-being

\author{
*Diego GarcíáÁlvarez, **Juan Hernández-Lalinde, ***María José Soler \\ *Universidad Rafael Belloso Chacín (Venezuela), **U niversidad Simón Bolívar (Colombia), ***A sociación Civil Jóvenes Fuertes
}

(Uruguay)

\begin{abstract}
Resumen. El enfoque del desarrollo positivo adolescente estácentrado en el estudio de los recursos, activos y factores de orden interno y externo que promueven la salud mental, el adecuado funcionamiento psicosocial, el florecimiento y una transición exitosaalaadultez. La escala anal izadaen este trabajo se sustentaen este constructo y busca plantear unaalternativa de medición paralosfactores promotores del desar rollo de dichaetapaevolutiva. El objetivo de lainvestigación fueevaluar las propiedades psicométricas del instrumento propuesto para tal fin por García-Álvarez y Soler, inspeccionando aspectos como lavalidez factorial, convergente y discriminante, pero también atributos como la invarianza de mediday la consistencia interna. Asimismo, se trazó como propósito determinar el rol predictivo de los activos del desarrollo adolescente sobreel bienestar psicológico. Paraello, se contó con la participación de 257 adolescentesde M ontevideo, U ruguay, con edadescomprendidas entre 12 y 19 años, en un estudio instrumental, transversal, correlacional y no experimental. Los resultadosindican que la propuesta original de los autores tiene fallas de validez factorial, convergente y discriminante, razón por la cual se planteó una escala alternativa compuesta por 12 ítems obtenidaal eliminar los reactivos problemáticos del cuestionario inicial. Losfactores resultantes de dicho instrumento fueron agrupados en autoestima, autoeficacia, autocontrol relacional, optimismo, percepción de riesgos y bienestar. Laversión abreviadareportó propiedades psicométricas apropiadas en todos losniveles evaluados, incluso en lo referente alainvarianzafactorial. Los hall azgos también dan cuenta del efecto predictor de estosfactores sobre el bienestar psicológico y sus dimensiones: control, aceptación, víncul os y proyectos. Se concluye que la opción reducida de la escala de los factores del desarrollo adolescente es una alternativa váliday confiable que puede ser utilizada como herramienta de medición de esta variable en la adolescencia temprana, tanto en el campo de intervenciones psicológicas como en el de investigación.
\end{abstract}

Palabrasclave: desarrollo positivo adolescente, factorespromotoresdel desarrollo, bienestar psicológico, estudiosde validación, adolescentes.

\begin{abstract}
The approach to positive adolescent development is centered on the study of resources, assets, and inter nal or external factors that promotemental health, adequate psychosocial functioning, flourishing and asuccessful transition to adulthood. The scale analyzed inthis work is based on this construct and seeksto propose a measurement alternative for the factors that promotethe development of this evolutionary stage The research aim wasto evaluate the psychometric properties of theinstrument proposed for thispurpose by García-Álvarez and Soler, inspecting aspects such as factorial, convergent and discriminant validity, but al so attributes such as measurement invariance and internal consistency. Likewise, the purpose wasto deter mine the predictive role of adolescent development assets on psychological well-being For this end, 257 adolescents from M ontevideo, U ruguay, aged between 12 and 19 years old, participated in an instrumental, cross-sectional, correlational and non-experimental study. The resultsindicate that the original proposal of the authors hasflaws in factorial, convergent and discriminant validity, which is why an alternative scale composed of 12 items was proposed, obtained by eliminating the problematic items from the initial questionnaire. The resulting factors of this instrument were grouped into self-esteem, self-efficacy, relational self-control, optimism, perception of risks and well-being The short version reported appropriatepsychometric properties at all levels eval uated, including factor invariance. The findings also account for the predictive role of these factorson psychological well-being and its dimensions: control, acceptance, attachment and projects. It isconcluded that the reduced option of the adolescent development factors scale eisavalid and reliable alternative that can be used as a measurement tool for this variable in early adolescence, both in the field of psychological interventions and in research.
\end{abstract}

Keywords: positive adolescent development, development promoting factors, psychological well-being, validation studies, adolescents.

\section{Introducción}

La adolescencia es una etapa evolutiva entre la niñez y la adultez caracterizada por ser una construcción social influidapor determinantes biológicos, socioculturales e históricos que imprimen retos, oportunidades de crecimiento y también riesgos psicosociales. En este

Fecha recepción: 11-09-20. Fecha de aceptación: 13-12-20

Juan Diego Hernández Lalinde

j.hernandezl@unisimonbolivar.edu.co sentido, la O rganización M undial de la Salud (OMS) a través de la O rganización Panamericana de la Salud (OPS) hizo un llamado expreso en 2018 a acelerar estudios que profundizaran en la salud mental adolescente debido a que son un grupo etario expuesto a múltiples enfermedades de causas prevenibles. Asimismo, se planteó que las personas adolescentes son actores con un rol activo y constructivo en sus procesos de salud; en efecto, las intervenciones deben ir orientadas a promover comportamientos saludables, positivosy adaptativos que permitan reducir múltiples factores de riesgo. Lo ante- 
rior, en conjunto con la exhortación de la O MS de comprender ampliamente el bienestar psicosocial, ha derivado en programas como Life Skills. Esto constituye una evidencia de los esfuerzos emprendidos en el área del desarrollo positivo adolescentey de la salud mental como vías de intervención basadas en evidencias.

El PositiveYouth Development es un enfoque teóricopráctico centrado en el estudio de la transición exitosa hacia la adultez, el cual está configurado en competencias adolescentes que posibilitan la adecuada adaptación a las distintas demandas de la etapa evolutiva, concibiendo la sal ud adolescente como un estado óptimo que va más allá de la ausencia de problemas y de sintomatología clínica. Al respecto, Lerner et al ., (2009) concibieron un modelo denominado las cinco ces, cinco constructos que hacen referencia a indicadores que viabilizan una adolescencia sana, floreciente y con una adaptación satisfactoria a las tareas psicosociales. Estos constructosson lacompetencia, conexión, carácter, confianza y compasión. Por otro lado, el modelo de las fortalezas personales formulado por Benson et al., (2006) está basado en una variedad de factores 0 activos del desarrollo que sostienen el desarrollo positivo en la adolescencia y están constituidos por 20 recursos externos y 20 internos. Para este enfoque, los activos del desarrollo promueven competencias adolescentes e incluso sirven como protectores ante factores y conductas de riesgo, mientras que el florecimiento ocurre cuando los activos internos del desarrollo se al inean con los recursos externos del contexto (O liva et al., 2020).

En la actualidad iberoamericana, el enfoque salutogénico de la adolescencia se denomina Desarrollo Positivo Adolescente (DPA) y se entiende como un modelo con raíces sistémicas, biológicas, contextuales, ecológicas y sociocomunitarias, respaldado en la multidisciplinariedad y en la promoción universal del crecimiento psicológico mediante la creencia en el potencial para el cambio intrapersonal de la mano de la plasticidad del desarrollo evolutivo, a pesar de las dificultades vinculadas al entorno (0 liva et al., 2017).

Deahí que, en lapraxis profesional dirigida ala atención psicológica, los modelos de prevención de riesgos psicosociales y de promoción de la salud mental no son antagónicos; por el contrario, son esfuerzos que se complementan y se relacionan íntimamente mediante la identificación de factores protectores conocidos como activos del desarrollo adolescente dentro del DPA (0 liva et al., 2017). Por tanto, las intervenciones diseñadas desde este enfoque pueden tener correlatos en varios niveles de atención psicológica; a saber: (a) promoción de la salud orientada al reconocimiento de fortalezas personales, activos y competencias; (b) prevención primaria concentrada en cambiar hábitos pocos sal udables, así como en la atención de síntomas comunes presentes en poblaciones expuestas a factores de riesgo como la violencia, consumo de alcohol u otras sustancias, entre otras situaciones; y (c) prevención secundaria a través de estrategias de atención psicológica específicas como la orientación, counseling y psicoterapia centrada en el desarrollo de activos y competencias personales, a la par del tratamiento clínico motivo de consulta (Alves, do Carmo Eulalio, \& Brobeil, 2009; Gallegos, 2017).

Entonces, el DPA es un constructo complejo que concibe una adolescencia centrada en competencias 0 recursos psicológicos (Benson et al. , 2006) que permiten latransición a la adultez de manera adaptativa, orientadaal Bienestar Psicológico (BP) y al desarrollo de competencias, fortalezas o recursos (O liva, 2015). El DPA habilita abordajes en diferentes niveles de atención y psicoeducación, siendo uno de sus ejes centrales los activos del desarrollo. Estos puedes ser conceptualizados como recursos personales, escolares, comunitarios, contextuales y familiares que promueven el DPA, Ilegando incluso a ser considerados factores promotores del desarrollo debido a que actuarían como catalizadores positivos (O liva et al., 2010). En este trabajo se hace énfasis en los factores promotores del desarrollo a nivel personal o interno; es decir, en aquellas características, competencias y estados de la personalidad que se vinculan con el desarrollo positivo y al funcionamiento psicológico óptimo en la adolescencia(Scales, Benson, Leffert, \& Blyth, 2000).

Cabe destacar que la escuela como institución tiene un papel protagónico en la promoción de la salud mental debido a que es un espacio donde confluyen políticas públicas y otros múltiples effuerzos abocados al desa rrollo integral de la personalidad de estudiantes en forma de programas extraescolares, talleres o espacios de crecimiento (Antolín-Suárez, O liva, Pertegal-Vega, \& Jiménez, 2011; Cobo \& García, 2017). En los últimos años, se han incrementado las intervenciones en contextos educacionales por medio de programas con resultados interesantes en áreas de funcionamiento psicosocial estudiantil. Ejemplos de ello son creciendo fuertes (García-Álvarez \& Soler, 2020; García-Álvarez, Soler, \& Cobo, 2020), aulasfelices (Lombas et al. , 2019), gimnasio de fortalezas (Proctor et al., 2011; Proctor et al., 2015) y otros específicos en la promoción del DPA en ámbitoseducativos como los propuestos por Catalano et al., (2019). 
Ahora bien, el DPA ha sido operacionalizado por distintos investigadores de manera similar, coincidiendo en identificar alrededor de 40 activos externos e internos (Benson, 1996; Leffert et al., 1998). A nivel internacional se han desarrollado escalas clásicas, una de ellas es la propuesta por el Search Insitute conocida como Profiles of Student Life: Attitudes and Behaviors (Leffert et al. , 1998) constituida por 160 ítems aglomerados en 16 factores con indicadores cuestionables de confiabilidad y validez. Este instrumento ha sido desaconsejado para su uso en evaluaciones de programas, por lo que más tarde el mismo Search Institute (2005) propuso el Devel opmental Assets Profile (DAP) integrado por 58 ítems reunidos en ocho dimensiones que explicarían las fortalezas del desarrollo adolescente, cuyas adecuadas propiedades psicométricas han permitido que se proponga incluso para investigaciones longitudinales. Benson et al., (2011) consideran que este cuestionario es el único sugerido para la evaluación en diseños pretest-postest. Además, Scales (2011) refirió que el DAP exhibió buenas propiedades psicométricas en cinco muestras internacionales, aunque también señaló dificultades en las versiones traducidas en lo referente a la validez cultural.

En la práctica, tales escalas tienen el inconveniente de ser excesivamente largas, si bien su capacidad de abarcar permite que sean utilizadas como inventarios. No obstante, otros autores han propuesto instrumentos menos extensos orientados a medir únicamente algunos de estos factores (Betancourt-0 campo, AndradePalos, \& Marín-M artínez, 2019). Ejemplos se hallan en el Adolescent $H$ ealth and Behaviors Survey de Reininger et al. (2003) que mide activos del desarrollo en adolescentes embarazadas, o también en el Youth Asset Survey de 0 man et al., (2002). 0 tras son las escalas para medir activos del desarrollo en el barrio de O liva, AntolínSuarez y López (2012), así como el cuestionario que evalúa for tal ezas y activos escolares desde la perspectivadel profesorado formulado por Pertegal-Vega, Gómez y 0 liva (2011), o la escala propuesta por Antolín-Suárez et al., (2011) que analiza los valores para el desarrollo positivo adolescente.

Para el diseño, ejecución y evaluación de intervenciones en el campo de la educación emocional, del carácter y del bienestar basados en el DPA y la psicología positiva como aliadadel sistemaeducativo (H osseinabad, Najafi, \& Rezaei, 2019; W hite $\&$ Waters, 2015), se hace necesario contar con escalas psicométricas val idadas que midan los activos internos del desarrollo en adolescentes educados, estimulados, promovidos o fortalecidos por cuenta de tales programas. Una exploración de la literatura psicométrica en U ruguay refleja la inexistencia de escalas que val oren Factores del Desarrollo Adolescente (FDA) desde una perspectiva centrada en el DPA, en la psicología positiva y en la salud mental. La única excepción la constituye el instrumento ADA que explora síndromes con base en una técnica de cribado centrada en la salud mental como ausencia de psicopatologías y que solamente incluye la dimensión de prosocial idad y resiliencia como aproximación al adecuado funcionamiento del individuo (D aset et al. , 2015; Fernández et al., 2020).

En este contexto, los autores del presente trabajo se adscriben a la sugerencia de crear escalas propias que se correspondan con las características socioculturales y psíquicas de la población adolescente montevideana en vez de adaptar instrumentos foráneos como los anglosajones o europeos (Carretero-Dios \& Pérez, 2005). Además, los autores aspiran a generar una escala breve que pueda ser empleada por investigadores de la región que se interesen en documentar el efecto de intervenciones para la promoción del DPA. Para ello, se tomó como punto de partida la literatura especializada que hace énfasis en la construcción de modelos del desarrollo positivo como el propuesto por O liva et al., (2010, 2015). En este, se listan 27 competencias ordenadas de mayor a menor importancia para el florecimiento en la adolescencia, las cuales, a su vez, se agrupan en cinco grandes áreas: social, cognitiva, emocional, moral y desarrollo personal.

Sin embargo, de esta extensa lista de activos del desarrollo se seleccionar on los más acordes ala real idad sociocultural montevideana, aquellos que pudieran ser comunes en los distintos programas de psicología positiva o los que se empleen para promover la salud mental y el DPA en contextos educativos para la adolescencia temprana. De manera específica, se escogieron los activos de autoestima, autoeficacia, optimismo y orientación al futuro, concibiéndolos como atributos personales que pudieran activarse en programas de intervención con adolescentes. Por otro lado, y como señala O liva (2015), los activos del desarrollo no solo capitalizan las competencias individuales, sino que también buscan proteger ante al gunos problemas comunes de la adolescencia; esto es, aquellos peligros psicosociales que afectan a las personas en esta edad temprana. En consecuencia, para la concepción de los factores del desarrollo también se tomaron en cuenta los asociados con la reducción y prevención de conductas de riesgo psicosocial 0 de percepción de riesgo. 
En este trabajo de investigación se asume que los esfuerzos para la promoción de la salud pueden enmarcarse en el DPA a través de una educación emocional, enfocada en el fortalecimiento del carácter y bienestar como estrategia de prevención inespecífica. Esta aproximación pudiera tener efectos beneficiosos y actuar como barrera de protección ante los variados factores de riesgo a los que la adolescencia está actualmente expuesta (García \& Serrano, 2017; Cobo \& García, 2017; Bisquerra Alzina \& Hernández Paniello, 2017; Seligman, Ernst, Gillham, Reivich \& Linkins, 2009; Ruiz-Aranda, Cabello, Salguero, Castillo, Extremera \& Fernández-Berrocal, 2010). En tal sentido, este estudio se justifica puesto que los resultados obtenidos aportarían a otras experiencias científicas para la promoción del BP en ámbitos de educación formal, lo que iría en concordancia con los antecedentes uruguayos de GarcíáÁlvarez \& Soler (2020).

Por todo lo anterior, se plantea como objetivo general de la investigación validar una escala que pueda ser empleada en programas encaminados a mejorar la educación emocional, del carácter y del bienestar, instrumento que se sustente en la psicología positiva y en el desarrollo positivo de la etapa adolescente. Por consiguiente, se plantean los siguientes objetivos específicos: (a) determinar la validez factorial de la escala de los FDA propuesta por los autores; (b) establecer la validez convergente de este instrumento; (c) examinar la red nomológica entre los constructos que representan a los activos del desarrollo adolescente y al bienestar psicológico; (d) calcular la validez divergente del cuestiona rio; (e) estimar la confiabilidad de la mencionada medida; (f) determinar la invarianza configural, métrica, escalar y residual de la alternativa resumida; y $(\mathrm{g})$ explicar el rol de los FDA como posibles predictores de niveles elevados de BP.

\section{M étodo}

\section{Tipo de investigación}

La investigación se clasifica como instrumental (M ontero \& León, 2007) ya que se enfoca en establecer las propiedades psicométricas de la escala FDA. Asimismo, se considera transversal, no experimental y correlacional debido que evalúa el rol predictivo de los FDA sobre el BP (Hernández Sampieri, Fernández CoIlado, \& Baptista Lucio, 2014).

\section{Participantes}

El estudio contó con la participación de 257 adolescentes, los cuales vivían todos en la ciudad de M ontevi- deo, U ruguay. La selección se llevó a cabo a través de muestreo propositivo, contactando a los participantes en instituciones educativas de la mencionada capital. En función de que no se utilizó una técnica probabilística de muestreo, el tamaño de la muestra quedó determinado por las restricciones económicas, logísticas y de tiempo vinculadas al proyecto. Los criterios de inclusión y exclusión fueron los siguientes: (a) tener una edad comprendida entre 12 y 19 años; (b) ser estudiante de ciclo básico regular; (c) estar residenciado en M ontevideo; y (d) carecer de cualquier condición física, mental o emocional que impidiese la aplicación de los instrumentos 0 que alterase los resultados.

\section{Instrumentos}

La escala FDA se planteó como una alternativa para medir este constructo a partir de 23 reactivostipo Likert con opciones de respuesta que van desde 1 (totalmente en desacuerdo) hasta 5 (totalmente de acuerdo). Fue diseñada con la intención de medir solo algunos de los activos del desarrollo que pudiesen ser promovidos en programas de salud mental, Life Skills, psicología positiva y DPA, lo que a su vez permitiría contar con un instrumento que pudiera ser empleado para evaluar el efecto de intervenciones e incluso la correlación con otros constructos.

Los autores refieren que en la fase inicial se propuso una versión preliminar de los ítems luego de una extensa y profunda revisión de la literatura especial izada, enfocada, principalmente, en losfructíferos aportes teóricos de O liva (2015), O liva et al., (2010) y O liva et al., (2011a, 2011b). Así pues, la versión original del instrumento que constaba de 23 ítems mostró evidencias de validez de contenido, propiedad que fue analizada a través del juicio de ocho expertos en la temática, tanto nacionales como internacionales, quienes fueron requeridos para una evaluación cualitativa de criterios como representatividad, pertinencia, coherencia, claridad y legibilidad con el dominio teórico y nivel evolutivo adolescente. Posteriormente, en la prueba piloto realizada con 89 adolescentes tempranos se hallaron coeficientes de confiabilidad mediante el alfa de C ronbach de .93 en Io que respecta al constructo total. Durante la administración del cuestionario se comprobó que los reactivos fuesen comprensibles para el grupo etario analizado.

Esta versión fue empleada por primera vez como escala ad hoc para inspeccionar el desempeño del programa creciendo fuertes (GarcíaÁlvarez \& Soler, 2020). El sentido funcional que los autores le dieron a la escala fue el de explorar factores del desarrollo adolescente 
desde la autopercepción, tras un programa basado en la promoción de las fortalezas del carácter de la psicología positiva con adolescentes en contexto educacional. Por un lado, se buscaba medir la promoción de los activos del desarrollo en adolescentes, específicamente aqueIlos que tienen que ver con la autoestima, autoeficacia, optimismo, orientación al futuro, satisfacción y bienestar; por otro, se pretendió val orar la percepción y prevención de conductas de riesgo psicosocial concernientes al relacionamiento social, autocontrol, consumo de drogas, alcohol o cigarrillos.

En este trabajo se busca examinar a profundidad las propiedades psicométricas de la escala FDA formulada por García-Álvarez \& Soler (2020). Para alcanzar este propósito, se administró también la escala de bienestar psicológico para adolescentes de Casullo (2002), medida diseñada a partir de la teoría de bienestar conforma da por las dimensiones de control, vínculos, proyectosy aceptación, la cual ha sido utilizada en estudios previos con muestras de adolescentes de M ontevideo, al canzando adecuadas cifras de fiabilidad de .79 para la escala completa, además de evidencias de validez factorial, convergente y discriminante (García-Álvarez, Hernández-Lalinde, Espinosa-Castro, \& Soler, 2020). Por lo tanto, el propósito de esto fue el de comprobar la validez convergente y propiciar la red nomológica de la escala.

\section{Procedimiento}

El trabajo de campo inició cuando se les explicó a los involucrados que la colaboración del adolescente era totalmente voluntaria, que no revestía riesgo al guno y no implicaba compensaciones monetarias 0 académicas. También se aclaró que, en caso de así desearlo, el participante podía retirarse del estudio en cualquier momento sin que ello generara repercusiones negativas personales 0 escolares. Se hizo hincapié en que la información sería manejada con confidencialidad y que los datos solamente se emplearían para fines investigativos. Finalmente, se diligenció el consentimiento informado por medio de las instituciones educativas de M ontevideo a través de las juntas directivas. Esto se llevó a cabo respetando los aspectos éticos, normativos y legales de la Asociación Americana de Psicólogos (APA) y del Ejecutivo uruguayo a través del Decreto 379/ 0081.

\section{Análisis estadístico}

El cumplimiento de la normalidad multivariada se evaluó a través de la prueba de Mardia (Kankainen,
Taskinen, \& 0 ja, 2004), mientras que la presencia de datos atípicos se identificó mediante las distancias de Mahalanobis (De Maesschalck, J ouan-Rimbaud, \& Massart, 2000; Trigueros, Aguilar-Parra, González-Santos, \& Cangas, 2019), encontrándose violaciones en ambas premisas. Posteriormente, se llevó a cabo un análisis de valores perdidos sobre la cantidad de casillas vacías dentro de cada variable, pero también a lo interno de cada adolescente. En el primer caso, la fracción de datos ausentes más alta fue de 3.63\% en los ítems 3, 6,8 y 13 del instrumento de BP. En el segundo, el porcentaje máximo de reactivos sin contestar por participante fue de 7.14\%, a excepción de lo que se observó en dosadolescentes, en los cual es lainformación faltante ascendió a $34.52 \%$ y $42.86 \%$. Considerando que esto se presentó solo en dos personas, se tomó la decisión de descartar estos registros y aplicar el contraste de Little (Li, 2013) al resto de la muestra para determinar si la pérdida de información respondía a razones puramente aleatorias. En vista de que esta suposición no fue rechazada ( $X^{2}=110.48, g=103, p=.289$ ), se optó por completar la base de datos a través de técnicas de imputa ción múltiple.

La alternativa a la escala original de GarcíaÁlvarez \& Soler (2020) se sustentó en un Anál isis Paralelo (AP) de Horn (Çokluk \& Koçak, 2016) con el percentil 95 como punto de corte para los autovalores ajustados, procedimiento que reveló la existencia de seis variables latentes. Tras esto, se realizó un Análisis Factorial Exploratorio (AFE) para tener una idea de la estructura que sería propuesta en el que se utilizaron mínimos residual escomo método deextracción y el modo oblimin paralarotación de lamatriz (Díaz-Leal, Blanco-O rnelas, Benítez-Hernández, Aguirre-Vásquez, \& CandiaLuján, 2017; Domínguez-Alonso, López-Castedo, \& Portela Pino, 2017). Como criterio de eliminación de ítems se adoptó que la carga factorial o comunalidad fuesen menores que .50. Los hallazgos de esta etapa se compara ron con lo postulado en la psicología positiva para formular esquemas con pertinencia teórica.

A continuación, se examinó la validez de constructo de los modelos por medio de unA nálisis Factorial Confirmatorio (AFC). Como estrategia de estimación se adoptó la de máxima verosimilitud con errores estándares robustos y estadístico de Satorra \& Bentler (2001). Aunque es una metodología que trabaja con va riables continuas, se justificó su uso debido a la ausencia de normalidad en una escala que cuenta con una estructura de cinco opciones (Bryant \& Satorra, 2012). Por otro lado, el tamaño muestral impidió laimplementación 
de métodos especialmente diseñados para manipular datos ordinales como el de mínimos cuadrados ponderadosen diagonal (Bandalos, 2014). Laadecuación delos modelos fue inspeccionada a través de la prueba chicuadrado y de la razón entre este estadístico y los gra dos de libertad. Se obtuvieron losíndices de ajuste comparativo (CFI), de Tucker-Lewis (TLI), de bondad de ajuste (GFI) y de bondad de ajuste corregido (AGFI), además de las raíces del error cuadrático medio por aproximación (RMSEA) y del residuo estandarizado medio (SRMR). Los puntos de corte que permiten cla sificar estos indicadores como aceptables u óptimos pueden consultarse en Bentler (1990) y se señalan en los resultados (Tabla 2).

La validez convergente se evaluó por medio del Índice de Fiabilidad Compuesto (IFC) y de la Varianza MediaExtraída (VME), en cuyo caso, los valores de referenciarespectivosfueron .60 y. 50 (Alarcón \& Sánchez, 2015). Para aportar mayores evidencias a la validez convergente y crear la red nomológica, se determinó la relación entre los FDA y el BP. La revisión teórica sugiere que la asociación entre estas variables debe ser positiva; sin embargo, considerando que son constructos afines, pero no iguales, se esperaría que la magnitud de las correlaciones sea moderada. En lo referente a la validez discriminante se implementó el método de Fornell-Larcker, según el cual esta validez queda esta blecidasi laVME es mayor que el cuadrado de las correlaciones entre cada factor y el resto de variables latentes (Fornell \& Larcker, 1981). También se utilizó el criterio de la razón de las correlaciones heterotrait-monotrait (HTMT). Esta metodología cuenta con una mayor sensibilidad para detectar la validez discriminante y la establece siempre y cuando sus valores sean menores que .80 (Henseler, Ringle, \& Sarstedt, 2015).

La fiabilidad de la escala y sus dimensiones se inspeccionó a través de los coeficientes alfa de Cronbach y omega de McDonald, utilizando el siguiente baremo para valorar la consistencia interna (Dunn, Baguley, \& Brunsden, 2014): cifras por debajo de .50, inaceptable; desde .50 hasta. 59 , pobre; desde .60 hasta. 69 , cuestionable; desde .70 hasta .79, aceptable; desde .80 hasta .89 , buena; y desde .90 hasta .95 , excelente. Con respecto a la confiabilidad de los ítems, se usó la media de la correlación ítem-total corregida, estadístico que se calculó al promediar la relación entre cada reactivo y el puntaje de la dimensión correspondiente excluyendo al elemento en cuestión. Este índice de homogeneidad se considera apropiado si su valor fluctúa entre . 30 y .70 (Wanous \& Hudy, 2001).
Con la finalidad de respaldar el uso de la escala de IosFDA para contrastar grupos, se examinó lainvarianza factorial de la propuesta abreviada. Se escogió esta versión del instrumento en vista de que fue la que reportó los mejores atributos psicométricos. Por otro lado, los gruposse formaron a partir de unavariable natural como el sexo, escogencia que se sustentó en diversos antecedentes que coinciden en señalar diferencias en el DPA entre varones y hembras (Scales et al., 2000). La invarianza se evaluó únicamente en las variables observables y considerando los cuatro niveles comunes: configural, examinando la estructura entre los grupos a través de un modelo sin restricciones; métrica, imponiendo la limitación de que las cargas factoriales fuesen iguales entre masculinos y femeninos; escalar, adicionando a la invarianza métrica la condición de igualdad en los interceptos; y estricta; en el que la invarianza de los residuos se añadió a las anteriores. La equivalencia multigrupos se verificó por medio de la prueba de la diferencia en los chi-cuadrados de los model os comparados, tomando como base el esquema configural. Sin embargo, debido a la sensibilidad de este estadístico al tamaño muestral, también se utilizaron los criterios que se enfocan en el incremento de índices de ajuste como el CFI y el RMSEA. En tal sentido, se ha propuesto que aumentos mayores que .01 0.015 en el CFI y en el RMSEA, respectivamente, implicarían el rechazo de la suposición de equivalencia (Cheung $\&$ Rensvold, 2002).

Para cumplir con el objetivo del estudio que se enfoca en la predicción, se plantearon modelos de regresión logística múltiple en los que las variables dependientes (VD) fueron las dimensiones de BP y las independientes (VI) fueron los FDA. Se prefirió esta opción ya que se incumplieron varios supuestos de la regresión lineal (Hernández-Lalinde, Espinosa-Castro, GarcíaÁlvarez, \& Bermúdez-Pirela, 2019; Hernández-Lalinde et al., 2018). Lospuntos de corte que permitieron transformar lasVD en dicotómicas se hallaron por medio de los cuartiles inferior y superior, de modo que la muestra se dividió en dos grupos con nivel alto y bajo de BP. Los FDA se ingresaron como regresores cuantitativos, pero también se utilizaron el sexo y la edad como covariables para obtener los odds ratio ajustados. El supuesto de linealidad fue evaluado mediante la prueba de Box-Tidwell sin encontrar inconsistencias, mientras que la multicolinealidad se descartó a través de los factores de inflación de la varianza y el índice de condición. Los datos atípicos fueron eliminados si sus residuos estudentizados superaban a 2 en valor absoluto, en tanto que la capacidad de predicción y el ajuste de regresión 
se sopesó con los pseudo $R^{2}$ de Cox-Snell, Nagelkerke y con los índices de sensibilidad y especificidad asumiendo como evento de interés el que el adolescente generara puntajes elevados de BP.

Finalmente, el procesamiento y análisis de los datos fue ejecutado con los programas SPSS (versión 25) y RStudio (versión 1.3.10.56). La significación de los resultados se fijó a partir de un tamaño de alfa de .05, salvo cuando se construyeron los intervalos bilaterales para el RMSEA, Ios cuales fueron obtenidos con un nivel de confianza de. 90.

\section{Resultados}

\section{Características sociodemográficas de los parti- cipantes}

La muestra estuvo conformada por 257 adolescentes, de los cuales, el $34.63 \%(n=89)$ fueron hembras y el $65.37 \%(n=168)$ fueron varones. La edad osciló desde 12 hasta 19 años, con media de $13.52 \pm 1.13$ ( $C V=8.36 \%)$. No hubo diferencias significativas en esta característica según sexo $(t=0.16, g l=255, p=.871)$, registrándose un promedio de $13.53 \pm 1.16(C V=8.57 \%)$ en los participantes masculinos y una media de $13.51 \pm 1.09$ ( $C V=8.07 \%)$ en las participantes femeninas.

\section{Planteamiento del nuevo modelo}

Tal y como se adelantó en la metodología, el AP reveló la existencia de seis factores subyacentes. Una

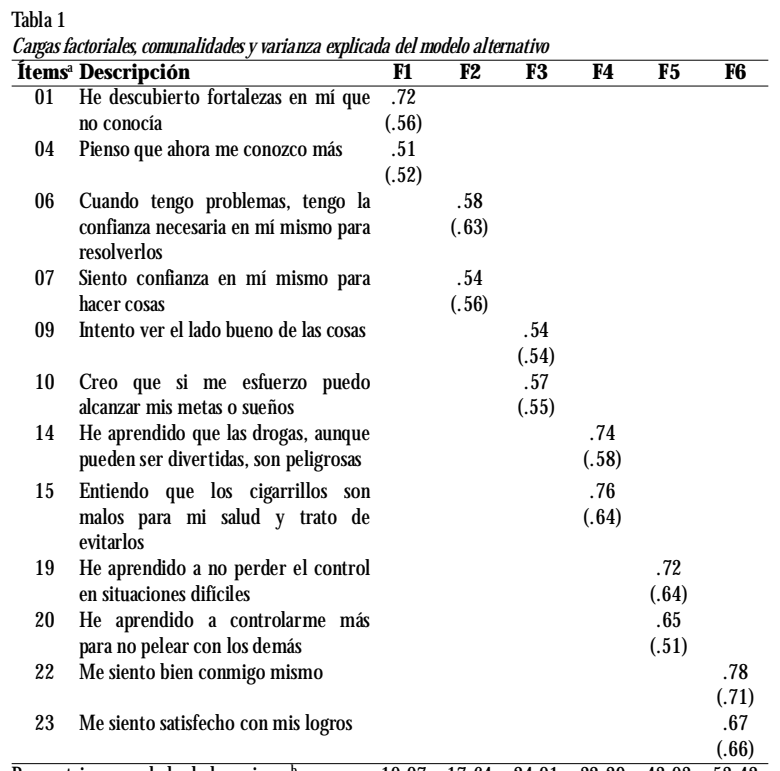
Fuente: elaboración propia. apara efectos de practicidad, se muestran únicamente los ítems cuya carga factorial estandarizada y cuya comunalidad fueron mayores o iguales que .50 . bEl porcentaje acumulado de la varianza explicada corresponde al AFE de los 23 ítems, aunque se muestren solo los 12 seleccionados. Abreviaturas: F1: autoestima. F2: autoeficacia. F3: optimismo. F4: percepción de riesgos relacionados con drogas y alcohol. F5: autocontrol relacional. F6: bienestar. vez dilucidado esto, se llevó a cabo unAFE con mínimos residuales para la extracción de factores y rotación oblimin para la obtención de la estructura más simple. La pertinencia de esto fue verificada a través de la medida de Kaiser-M eyer-O Ikin (KMO) y por medio de la prueba de esfericidad de Barlett, encontrándolo adecuado (KMO $\left.=.91, X^{2}=2652.71, g=253, p<.001\right)$. Tras esto, se eliminaron 11 de los 23 ítems originales, aquellos que incumplieron con la condición de que la carga estándar factorial y la comunalidad fueran mayores 0 iguales que .50. Los reactivos seleccionados fueron posteriormente revisados para comprobar su pertinencia teórica, lo que permitió formular el modelo alternativo que diera respuesta a los objetivos de la investiga ción (Tabla 1).

\section{Validez factorial}

El esquema original de 23 reactivos tuvo problemas a nivel factorial. Si bien es cierto que la razón $X^{2} / \mathrm{gl}$ resultó óptima y medidas como el RMSEA y SRMR fueron aceptables, otros índices como el CFI,TLI, GFI y AGFI arrojaron cifras que estuvieron por debajo de lo estipulado, incluso a nivel del mínimo admisible (Tabla 2). Por otro lado, la versión abreviada de 12 elementos gozó de un ajuste claramente superior; de hecho, todos los índices fueron óptimos a excepción de la significa ción de la prueba chi-cuadrado, cuya cantidad puede considerarse aceptable (Tabla 2).

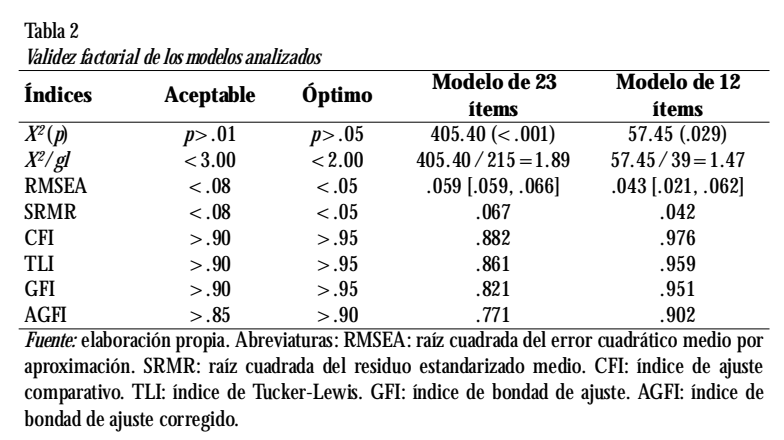

\section{Validez convergente y red nomológica}

En cuanto a la validez convergente, la escala de 23 ítems registró IFC satisfactorios; sin embargo, lasVME fueron menores que .50 en todas las dimensiones, menos en F2 (autoeficacia) y F5 (autocontrol relacional). Esto no se observó en la medida abreviada, en la que ambos criterios se cumplieron a cabalidad (Tabla 3). En lo referente a la red nomológica, las correlaciones se comportaron según lo esperado. 0 bsérvese que en ambas versiones del cuestionario los coeficientes fueron positivos, significativosy de magnitud moderada en su mayoría (Tabla 4). 
Validez convergente y confiabilidad de las escalas analizados

\begin{tabular}{lcccccccccc}
\hline \multirow{2}{*}{ Factores } & \multicolumn{3}{c}{ Escala de 23 ítems } & \multicolumn{4}{c}{ Escala de 12 ítems } \\
\cline { 2 - 11 } & VME & IFC & $\alpha$ & $\omega$ & CITC & VME & IFC & $\alpha$ & $\omega$ & CITC \\
\hline F1 & .48 & .82 & .82 & .82 & .61 & .50 & .66 & .66 & .67 & .49 \\
F2 & .62 & .76 & .76 & .76 & .62 & .62 & .76 & .76 & .76 & .62 \\
F3 & .48 & .66 & .65 & .65 & .49 & .53 & .66 & .64 & .68 & .48 \\
F4 & .43 & .75 & .74 & .75 & .54 & .58 & .73 & .73 & .73 & .57 \\
F5 & .69 & .82 & .82 & .82 & .69 & .69 & .82 & .82 & .82 & .69 \\
F6 & .34 & .80 & .80 & .80 & .51 & .64 & .78 & .77 & .78 & .62 \\
Total & .46 & .95 & .92 & .94 & .55 & .60 & .94 & .87 & .92 & .56 \\
\hline
\end{tabular}

uente: elaboración propia. A breviaturas: VME: varianza media extraída. IFC: índice de fiabilidad compuesto. CITC: promedio de la correlación ítem-total corregida. F1: autoestima. F2: autoeficacia. F3: optimismo. F4: percepción de riesgos relacionados con drogas y alcohol. F5: autocontrol relacional. F6: bienestar.

Tabla 4

Red nomológica entre los FDA y el B

\begin{tabular}{|c|c|c|c|c|c|c|c|c|c|c|}
\hline \multirow{2}{*}{ Factores } & \multicolumn{5}{|c|}{ Escala de 23 ítems } & \multicolumn{5}{|c|}{ Escala de 12 ítems } \\
\hline & C & V & $P$ & A & BPT & C & V & $P$ & A & BPT \\
\hline F1 & $.39^{* * *}$ & $.35^{* * *}$ & $.35^{* * *}$ & $.42^{* * *}$ & $51^{* * *}$ & $29^{* * *}$ & $.28^{* * *}$ & $.30^{* * *}$ & $.27^{* * *}$ & $.37^{7 *}$ \\
\hline F2 & $.37^{* * *}$ & $.28^{* * *}$ & $.32^{* * *}$ & $.33^{* * *}$ & $.40^{* * *}$ & $.37^{* * *}$ & $.28^{* * *}$ & $.32^{* * *}$ & $.33^{* * *}$ & $.40^{* * *}$ \\
\hline F3 & $.40^{* * *}$ & $.23^{* * *}$ & $.30^{* * *}$ & $.33^{* * *}$ & $.43^{* * *}$ & $.35^{* * *}$ & $.30^{* * *}$ & $.36^{* * *}$ & $.26^{* * *}$ & $.43^{* * *}$ \\
\hline F4 & $.34^{* * *}$ & $.35^{* * *}$ & $.36^{* * *}$ & $.27^{* * *}$ & $.44^{* * *}$ & $.16^{*}$ & $.18^{* *}$ & $.25^{* * *}$ & $.09 \dagger$ & $.21^{1 * *}$ \\
\hline F5 & $.36^{* * *}$ & $.37^{* * *}$ & $.30^{* * *}$ & $.44^{* * *}$ & $.47^{* * *}$ & $.41^{* * *}$ & $.25^{* * *}$ & $.28^{* * *}$ & $.35^{* * *}$ & $.42^{* * *}$ \\
\hline F6 & $.42^{* * *}$ & $.34^{* * *}$ & $.35^{* * *}$ & $.30^{* * *}$ & $.45^{* * *}$ & $.36^{* * *}$ & $.37^{* * *}$ & $.30^{* * *}$ & $.44^{* * *}$ & $.47^{* * *}$ \\
\hline Total & $.50^{* * *}$ & $.40^{* * *}$ & $.44^{* * *}$ & $.43^{* * *}$ & $.59^{* * *}$ & $.49^{* * *}$ & $.39^{* * *}$ & $.44^{* * *}$ & $.42^{* * *}$ & $.57^{* * *}$ \\
\hline
\end{tabular}

\section{Validez discriminante}

El criterio de Fornell-Larcker permite apreciar que la escala diseñada por los autores reporta inconvenientes de validez discriminante. Esta metodología confirma tal propiedad si laVME de cada factor es mayor que el cuadrado de las correlaciones con el resto de varia bles latentes (Fornell-Larcker, 1981). Como puede notarse (Tabla 5), tal condición se viola en repetidas ocasiones; por ejemplo, laVME de F1 (autoestima) fue menor que las correlaciones cuadráticas registradas en la columna correspondiente. Algo similar se advierte en F2 (autoeficacia), cuyaVME fue menor que la correlación al cuadrado entre F2 y F1. Para completar el aná lisis, repítase lo anterior sobre todas las filas y columnas relevantes que se localicen por debajo de la diagonal.

Por otro lado, compruébese (Tabla5) queen la adaptación reducida del instrumento solo se presenta esta inconsistencia cuando se coteja laVME de F1 con la correlación al cuadrado entre F1 y F5 (autocontrol relacional). Ahora bien, al respecto del segundo criterio, el cociente de las correlaciones HTMT se compara

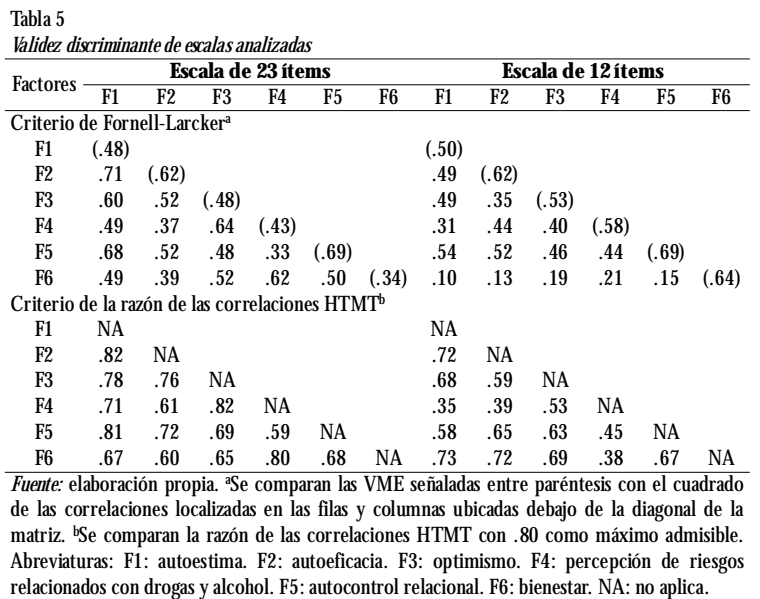

con puntos de corte para establecer la validez discriminante, la cual quedaría verificada si ninguno de estos valores fuese mayor que .80 (Henseler et al., 2015). Nótese (Tabla 5) que esta regla se incumple tres veces en la medida original de 23 afirmaciones, mientras que tal inconformidad no se presenta en laescala de 12 ítems.

\section{Confiabilidad}

En general, laconfiabilidad de ambas escal asfue apropiada. Como puede apreciarse (Tabla 3), la propuesta original cuenta con coeficientes alfa y omega mayores que . 70 en todas las dimensiones salvo en F3 (percepción de riesgos relacionados con drogas y al cohol). Esto se repite en laalternativa reducida, aunque en esta oportunidad tanto F3 como F1 (autoestima) reflejan índices menores que .70. Ahora bien, con respecto al promedio de la correlación ítem-total corregida (CITC), las dos propuestas cumplen con el criterio ya que todos los valores reportados oscilan desde 30 hasta. 70 (Tabla 3). En adelante, la descripción de los resultados se limitará solamente a la versión resumida del instrumento debido que dicha alternativa ha evidenciado las mejores propiedades psicométricas.

\section{Invarianza factorial de la escala abreviada se- gún sexo}

La invarianza de medida fue comprobada a todos los niveles. Verifíquese que el ajuste de la estructura configural fue satiffactorio, que la significación del test para la diferencia de todos los estadísticos chi-cuadrado fue mayor que 05 y que en ningún momento hubo incrementos en el CFI 0 en el RMSEA que superaran .01 0.015 (Tabla 6). Estos hallazgos avalan el uso del instrumento como herramienta para contrastar grupos según sexo.

\begin{tabular}{|c|c|c|c|c|}
\hline ndices & $\begin{array}{c}\text { Modelo }^{\text {c }} \\
96.44(077)\end{array}$ & $\begin{array}{c}\text { Modelo }^{\text {d }} \\
99.39(120)\end{array}$ & $\begin{array}{c}\text { Modelo }^{\text {e }} \\
105.34(129)\end{array}$ & $\begin{array}{c}\text { Modelo }_{4}{ }^{f} \\
119.07(119)\end{array}$ \\
\hline TI & 78 & 84 & 90 & 102 \\
\hline lodelo comparativo & NA & Modelo & ModelO $_{2}$ & $\mathrm{ModelO}_{3}$ \\
\hline$\Delta \mathrm{gl}$ & NA & 6 & 6 & 12 \\
\hline$\Delta X^{2}(p)$ & NA & $2.95(.815)$ & $5.95(.429)$ & $13.73(.318)$ \\
\hline MSEA & .043 & .038 & .036 & .0 \\
\hline RMSEA & NA & .005 & .002 & .000 \\
\hline $\mathrm{CFI}$ & .977 & .981 & .981 & .979 \\
\hline$\Delta \mathrm{CFI}$ & NA & .004 & .000 & .002 \\
\hline $\begin{array}{l}\text { Fuente: elaboración } \\
\text { bM odelo con cargas } \\
\text { factoriales e intercep } \\
\text { interceptos y residual } \\
\text { los grados de libertad } \\
\text { cuadrado entre el mo } \\
\text { por aproximación. SR } \\
\text { comparativo. } \Delta \text { CIF: }\end{array}$ & $\begin{array}{l}\text { aM odelo sin } \\
\text { iales iguales } \\
\text { ales para eval } \\
\text { ales para evalu } \\
\text { el model } o_{(i)} \text { y } \\
\text { y el model } o_{(i} \\
\text { ráz cuadrada }\end{array}$ & $\begin{array}{l}\text { tricciones } p \\
\text { evaluar inve } \\
\text { nvarianza es } \\
\text { ivarianza est } \\
\text { nodelo } o_{(i+1)} \\
\text { 2M SEA: raíz }\end{array}$ & $\begin{array}{l}\text { evaluar la inval } \\
\text { iza métrica. } \mathrm{M} \\
\text { r. dModelo con } \\
\text { a. A Abreviaturas: } \\
\text { diferencia en lo } \\
\text { adrada del error }\end{array}$ & $\begin{array}{l}\text { anza configural. } \\
\text { delo con cargas } \\
\text { argas factoriales, } \\
\text { gl: diferencia en } \\
\text { estadísticos chi- } \\
\text { uadrático medio } \\
\text { índice de ajuste }\end{array}$ \\
\hline
\end{tabular}

Predicción del BP a partir de los FDA utilizando la escala abreviada

Los FDA fueron predictores del BP y sus dimensio- 
nes en esta muestra de adolescentes montevideanos. Nótese (Tabla 7) que al menos dos activos del desar rollo reflejaron una asociación significativa con todos y cada uno de estos constructos. De manera concreta, obsérvese que, por ejemplo, la autoeficacia y el autocontrol relacional fueron predictores importantes de la dimensión de control adolescente, reportando los $O R$ ajustados que se señal an en los resultados (Tabla 7). Se podría interpretar en este caso que, al mantener constantes el resto de variables, un incremento de un punto en la autoeficacia aumenta 1.68 veces la probabilidad de generar valores elevados de control. Conclusiones similares se pueden derivar al analizar el efecto del autocontrol relacional sobre esta dimensión, pero también al revisar las demás subescalas del BP (Tabla 7).

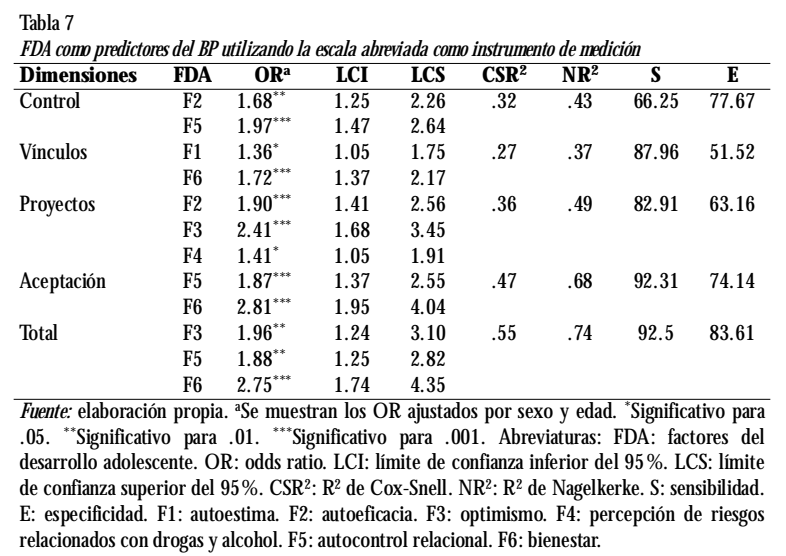

\section{Discusión}

Los resultados del AFE y AFC permitieron la reformulación del modelo original de los FDA; esto es, del instrumento hexafactorial original de 23 ítems se obtuvo una versión alternativa de 12 reactivos con adecuados indicadores de ajuste. Tomando como punto de partida los postulados teóricos del DPA, psicología positiva, psicología de la adolescencia, además de la correspondiente evidencia empírica, se planteó la estructura del nuevo esquema tal y como se señala a continua ción:

Factor 1: autoestima. Denominado de esta forma debido aque refiere al autoconcepto, autoconocimiento; al descubrimiento en el transcurso de las intervenciones de aquellas fortalezas en sí mismo que antes no se conocían. Tiene que ver también con la valoración que cada persona hace de sí mismo; en cuanto más se conozca e identifique sus fortalezas, más valoración, seguridad y estimatendráacerca de su yo (0 liva et al., 2011a).

Factor 2: autoeficacia. Dimensión que engloba las afirmaciones orientadas a medir la autopercepción adolescente en su capacidad para realizar tareas acordes a los objetivos, es decir, la confianza en sí mismo para realizar actividades e incluso para hacerle frente a problemas de diversa índole (Sánchez, 2014; O liva et al., 2011b).

Factor 3: optimismo. Activo del desarrollo que ma nifiesta la tendencia cognitiva a apegarse a estilos explicativos de la realidad más favorables; es decir, a creer que pueden suceder cosas según lo planificado mientras se toman acciones para ello. Implica estar dotado de orientación al futuro para viabilizar una organización conductual a la persona adolescente que le permita concretar acciones del presente hacia el porvenir (Sánchez \& M éndez, 2009; Scheier, Carver, \& Bridges, 1994).

Factor 4: percepción de riesgos relacionados con drogas y alcohol. Factor que agrupa ítems concernientes a la psicoeducación de riesgos psicosociales comunes a la etapa de la adolescencia. De manera específica, se concentra en el consumo de alcohol y drogas, advirtiendo que, aunque pudieran parecer divertidas, resultan peligrosas y nocivas para la sal ud integral del adolescente. Es una dimensión de suma importancia debido a que da cuenta del papel simultáneo de intervenciones dirigidas a prevenir y promover la salud en conjunto con losfactores descritosanteriormente (GarcíaÁlvarez $\&$ Soler, 2020).

Factor 5: autocontrol relacional. Reúne aquellos reactivos referidos a la competencia de regulación, incluyendo tanto ítems de orden intrapersonal como interpersonal. Estos se enfocan en la capacidad individual para reconocer y prevenir conductas riesgosas en lo psicosocial, evitando la pérdida de control en situaciones difíciles que podrían devenir en conflictos o peleas con sus pares (O liva, 2015).

Factor 6: bienestar. Congrega dos afirmaciones asociadas al nivel de satisfacción y bienestar mediante los cuales la persona adolescente hace una valoración de su vida. Es un factor relevante para el impulso de intervenciones en el área del DPA y de la psicología positiva en general, así como para la educación emocional, del carácter y para el bienestar. Esta dimensión favorecería la potenciación de la satisfacción y del bienestar psicológico como protectores expresados en un adecuado funcionamiento psicosocial, lo que a su vez redundaría en un desarrollo sano y unatransición óptimahaciala adultez (Casullo, 2002; Reina, Oliva, \& Parra, 2010; Ryff, 2014).

Esta nueva configuración de la escala FDA en su versión de 12 ítems, reunidos en dos elementos por cada factor, constituye un instrumento corto y de breve apli- 
cación que cuenta con excelentes propiedades psicométricas. En términos de confiabilidad, la medida presenta indicadores adecuados de consistencia interna evaluados integralmente a través de los coeficientes alfa y omega. También ostenta índices de homogeneidad apropiados medidos con el promedio de la CITC. Las cifras obtenidas en cuanto al IFC y a laVME también fueron aceptables, aportando evidencias para la validez convergente (Moral, 2019). La validez discriminante fue satisfactoria, explorada por medio de la razón de las correlaciones HTMT (Henseler et al., 2015) y del criterio de Fornell-Larcker (1981). Debido a lo anterior, se sugiere su uso para explorar factores del desarrollo adolescente entendidos como activos internos y componentes del desarrollo positivo adolescente.

Con base en lo anterior, la escala FDA puede ser considerada como una alternativa preferible a la Profiles of Student Life:Attitudes and Behaviors (Leffert et al., 1998) para evaluar los activos internos del desarrollo adolescente en M ontevideo en virtud de las numerosas críticas que ha recibido en términos de validez. Por otro lado, la medida DAP (Scales, 2011) ha ostentado indicadores de confiabilidad en factores como los valores positivos e identidad, que son los que más pueden asemejarse a los activos internos del desarrollo, que van desde .60 hasta .85 para el primero y desde .51 hasta .79 en el segundo. Estos resultados corresponden a estudios realizados en cinco países distintos y resulta interesante que la fiabilidad disminuye en lugares como Filipinas, Bangladés y Albania.

Desde luego, lo que se procuró desde el comienzo fue ofrecer una opción psicométrica que respondiera a necesidades de aplicación que fuesen económicas en tiempo y recursos en comparación con los antecedentes clásicos ya mencionados. La idea no fue en ningún momento la de proponer un inventario amplio de todos los activos del DPA, sino más bien la de formular una escala breve que permitiera evaluar los principales FDA, tanto en activos como en componentes o variables de ajuste adolescente, especialmente de los más comunes y de aquellos que pudieran ser objeto de intervención con base en muestras de adolescentes tempranos. Para los autores, tales factores son la autoestima, autoeficacia, optimismo, autocontrol relacional, percepción de riesgos, satisfacción y bienestar.

En lo que respecta a este trabajo, la principal venta jade laescalaFDA radica en el sentido práctico de medir factores internos que ayudarían a promover otras competencias, a saber, percepción de riesgos relacionados con drogas y alcohol, satisfacción y bienestar adolescen- te, e incluso otros recursos externos que posibilitarían el ajuste adolescente. A juicio de los autores, esta escala es una aportación necesaria y relevante dentro del campo de la promoción de la salud mental en esta etapa evolutiva, así como para el enfoque de investigación del desarrollo positivo adolescente. La ventaja de la escala es que permite evaluar las áreas comunes del desarroIlo de las competencias adolescentes, siendo las personales las que nutren al resto de competencias con correlatos externos y en distintos contextos como el escolar, familiar y relacional (O liva et al., 2020). Sería interesante el uso de alguna subescala de acuerdo con losobjetivos deintervencioneso investigaciones; en otras palabras, podría ser un recurso metodológico a ser empleado en evaluaciones psicológicas. También se recomiendan realizar mediciones longitudinales para explorar la estabilidad temporal, lo que, junto con las evidencias presentadas en esta investigación, pudiesen sugerir su empleo para evaluaciones de programas con diseño pretest-postest.

Asimismo, Benson (1997) y Scal es et al., (2000) explicaron que los FDA pueden tener efecto directo e indirecto sobre otros indicadores de funcionamiento psicosocial, siendo que al gunos de estos activos pueden ser capaces de catalizar y promover otros factores del desarrollo que conducen al florecimiento adolescente. Un ejemplo de ello se encuentra en aspectos internos como la autoestima y la autorregulación, las cuales sugieren que podrían existir núcleos de factores que conformen el desarrollo adolescente. De igual modo, 0 liva et al., (2020) en su más reciente modelo de desarroIlo positivo adolescente basado en una mirada de enfoque de género realizado con metodologías cuantitativas, han determinado que los activos personales del desarrollo como autoestimay autocontrol se asociaron con una menor sintomatología depresiva, con mayor bienestar y satisfacción, promoviendo aspectos como la sa lud mental y el ajuste adolescente.

A hora bien, la asociación entre los FDA y una medida externa como el BP dan cuenta de la red nomológica del constructo. En tal sentido, las correlaciones fueron positivas, significativas y con magnitud que fluctuó desde débil hasta fuerte, aunque en su mayoría fueron moderadas. Al ordenar de mayor a menor los coeficientes de Spearman-Brown encontrados se tiene que el tamaño de la relación con el bienestar psicológico fue con el autocontrol relacional, optimismo, percepción de riesgos vinculados al uso de drogas y alcohol, autoeficacia, autoestima y bienestar (García-Álvarez, Soler, \& Rendón, 2019). En esta mismalínea, los hallaz- 
gos emanados de los modelos de regresión logística fueron congruentes con antecedentes en los que se señala a los FDA como predictores de un adecuado funciona miento psicosocial (Mazloomy, Atabay, Rahimi, Fallahzadeh, \& Vaezi, 2019; Scales et al., 2000; Soares, Pais-Ribeiro, \& Silva, 2019). En esta ocasión, todos los activos del desarrollo se vieron involucradosal momento de pronosticar puntajes elevados de BP y de sus respectivas dimensiones. Compruébese, por ejemplo, que la autoeficacia y el autocontrol relacional tuvieron un efecto predictor importante sobre el control adolescente; es decir, a mayores puntuaciones en estos factores, más alta será la probabilidad de que el adolescente genere valoraciones elevadas en dicha dimensión, la cual, según Casullo (2002), manifestaría una capacidad prominente de dominar ciertas situaciones, así como de engrandecer la autocompetencia y la habilidad para aprovechar las oportunidades que brinde el entorno.

Asimismo, adolescentes con autoestima y bienestar en su perfil de FDA van a tener mayor probabilidad de tener puntuaciones el evadas en la dimensión víncul os de bienestar. Esto es esperable, pues en la adolescencia las relaciones con pares toman un importante lugar en el funcionamiento psicosocial. Para esto, a persona adolescente debe sentirse satisfecha y segura de sí misma a fin de confiar en paresy tener relaciones de calidad afectiva. Al mismo tiempo, los factores de autoeficacia, optimismo y percepción de riesgos ayudan a predecir altos puntajes en la dimensión de proyectos de bienestar, lo que permitiría una adecuada orientación al futuro y la creencia que se tiene en sus capacidades, en que las cosas van a suceder según las metas, objetivos y el sentido que se lleva en la vida. Por su lado, la dimensión aceptación se ve predicha por factores del desarrollo como autocontrol relacional y bienestar; es decir, en la medida en que la persona adolescente mejora sus competencias de regulación y satisfacción, en esa medida posibilita la aceptación de sí mismo y del entorno (Casullo, 2002; Ryff, 2014), hallazgos que tienen implicaciones prácticas para vías de intervención.

A pesar de las excelentes propiedades psicométricas a nivel de funcionamiento interno y externo de la escala de los FDA, esta investigación tiene limitaciones que invitan a ser cauteloso al momento de interpretar los resultados. La primera tiene que ver con el tamaño muestral, en este caso, relativamente pequeño. Además, el muestreo intencional llevado a cabo limitaría ostensiblemente la capacidad para generalizar los ha llazgos, aspecto que podría ser mejorado en futuros proyectos en los que se implementen técnicas aleatorias con grupos más numerosos y que incluyan participantes de distintas zonas de Montevideo en un esfuerzo por incrementar la representatividad del estudio. Asimismo, y a modo de recomendación, sería interesante estudiar la factibilidad de aplicar las dimensiones de la escala como subpruebas para medir FDA específicos. Por ejemplo, se podría valorar la autoeficacia a partir de una medida compuesta únicamente por dos ítems, tendencia que cada vez está siendo más demandada por el hecho de economizar, agilizar y abreviar la administración de instrumentos de evaluación psicológica (Domínguez-Lara, 2018; Domínguez-Lara, NavarroLoli, \& Prada-Chapoñán, 2019).

O tra sugerencia sería la de expandir la validez externa de la escala ampliando la red nomológica al incluir otros constructos de la salud mental adolescente que se enmarquen en la perspectiva del adecuado funcionamiento psicosocial. En este orden de ideas, se propone incluso evaluar la capacidad predictiva con varia bles psicopatológicas como la ansiedad, depresión y malestar. De igual manera, sería pertinente estudiar los factores del desarrollo adolescente de esta escala en consideración a la edad adolescente, así como desde la perspectiva de género y sexualidad.

\section{Conclusiones}

Se concluye que la escala FDA es una alternativa válida, breve y confiable para medir de forma económica un conjunto de activos del desarrollo de manera integral; a saber, autoestima, autoeficacia, optimismo, autocontrol relacional, percepción de riesgos, satisfacción y bienestar. La medida se recomienda especialmente para ser utilizada en intervenciones basadas en el DPA y en las distintas modalidades de la psicología positiva destinadas a la adolescencia temprana. En definitiva, en esta investigación la escala FDA presenta adecuadas evidencias de validez factorial, convergente, discriminante, invarianza y confiabilidad. Además, los factores señalados tienen capacidad predictiva sobre el BP y sus dimensiones, siendo indicadores de aproximación al funcionamiento psicosocial óptimo y a la salud mental.

\section{Agradecimientos}

Proyecto de investigación financiado por la asocia ción Jóvenes Fuertes U ruguay. Los autores desean expresar su profundo agradecimiento a cada adolescente, quienes dispusieron solícitamente de su tiempo para 
participar en el estudio y responder a los instrumentos correspondientes. También agradecer al equipo de tra bajo de la asociación civil Jóvenes Fuertes por su cola boración y por facilitar el contacto entre las diferentes instituciones educativas de M ontevideo y los investigadores. Sin su ayuda, la culminación de este proyecto no habría sido posible.

\section{Referencias}

Alarcón, D., \& Sánchez, J.A. (2015).Assessing convergent and discriminant validity in theADHD-R IV rating scale: U serwritten commands for AverageVariance Extracted (AVE), CompositeReliability (CR), and Heterotrat-M onotraitratio of correlations(HTMT). Spanish STATA M eeting, 39.

Alves, R. F., do Carmo Eulalio, M., \& Brobeil, S.A.J. (2009). La promoción delasaludylaprevención deenfermedadescomo actividades propias de la labor de los psicólogos. Arquivos BrasilerirosdePsicologia, 61(2), 1-12.

Antolín-Suárez, L.,O liva,A., PertegalVega, M,\&Jiménez,A. (2011). Desarrolloy validación deunaescalade valoresparael desa rrollo positivo adolescente. Psicothema, 23(1), 153-159.

Bandalos, D. L. (2014). Relative performance of categorical diagonally weighted least squares and robust maximum likelihood estimation. Structural Equation Modeling: A MultidisciplinaryJournal, 21(1), 102-116. doi.org/ 10.1080/ 10705511.2014.859510.

Benson, P. L. (1996). D evelopmental AssetsAmong M inneapolisouth: TheU rgency of Promoting H ealthy Community. M inneapolis Search Institute.

Benson, P. L., Scales, P. C., \& Syvertsen, A. K. (2011). The contribution of thedevelopmenta assetsframework to positive youth development theory and practice. In Advancesin child development and behavior (v. 41, pp. 197-230). IAI

Benson, P. L., Scales, P. C., Hamilton, S. F., Sesmal r,A., Hong, K. L., \& Roehlkepartain, E.C. (2006). Positiveyouth development so fa: Corehypotheses and their implicationsfor policy and practice. Search Insitutel nsights\& Evidence, 3(1), 1-13.

Bentler, P. M. (1990). Compartivefitindexesinstructural models Psychological Bulletin, 107(2), 238-246. doi.org/10.1037/ 0033-2909.107.2.238.

Betancourt-0 campo, D.,Andrade-Palos, P., \& Marín-M artínez, A. (2019). Análisispsicométrico delaversión revisadadela escaladefortalezasinternasy externasparaadolescentes(FIEAR). Revista Colombiana deCienciasSociales, 10(1), 20-39.

BisquerraAlzina, R. (2010). Educación Emocional y Bienestar. BarceIona. España: Edit. Praxis.

BisquerraAlzina, R., \& HernándezPaniello, S. (2017). Psicología positiva, educación emocional y el programa aulas felices. Papelesdel Psicólogo, 38 (1), 58-65.

Bryant, F. B., \& Satorra,A. (2012). Principlesand PracticeofScaled
DifferenceChi-SquareTesting Structural Equation M odeling:A Multidisciplinary Journal, 19(3), 372-398. doi.org/ 10.1080/ 10705511.2012.687671.

Carretero-Dios, H., \& Pérez, C. (2005). N ormasparael desarrolloyrevisión deestudiosinstrumentales. International Journal of Clinical and $H$ ealth Psychology, 5(3), 521-551.

Casullo, M. M. (2002). Evaluación del bienestar psicológico. En Casullo, M. M. (Comp.). (2002). Evaluación del bienestar psicológico en Iberoamérica (pp. 11-29). BuenosAires: Paidós.

Catalano, R. F., Skinner, M. L.,Alvarado, G., Kapungu, C., Rearley, N., Patton, G. C., ... \& Sawyer, S. M. (2019). Positiveyouth developmentprogramsin low-and middleincomecountries: aconceptual frameworkand systemticreview of efficacy.journal of Adolescent H ealth, 65 (1), 15-3.

Cheung, G.W., \& Rensvold, R. B. (2002). Evaluatinggoodness-offit indexes for testing measurement invariance. Structural Equation M odeling, 9(2), 233-255. doi.org/ 10.1207/ S15328007SEM 09025.

Cobo, R. \& García, D. (2017). Lafelicidadeseducable: apropósito del bienestar en lasescuelas. Revista Convocación, 33 -34, VII, 38-47.

Çokluk, Ö ., \& Koçak, D. (2016). Using H orn'sParallelA nalysis Method in Exploratory FactorAnalysisfor Determining the Number of Factors. Educational Sciences.Theory and Practice, 16(2), 537-551.

Daset, L. R., Fernández-Pintos, M. E., CostaBall, D., López-Soler, C., \&Vanderplasschen,W. (2015). Desarrolloy validación del autoinformedeadolescentes:ADA. CienciasPsicológicas, 9(1), 85-104.

DeM resschalck, R., Jouan-Rimbaud, D., \& M assart, D. L. (2000). TheM ahalanobisdistance. Chemomericsand IntelligentLaboratory Systems, 50(1), 1-18. doi.org/ 10.1016/ S01697439(99)00047-7.

Díaz-Leal,A. C., Blanco O rnelas, L. H., BenítezHernández,Z.P., AguirreVásquez, S. I., \&CandiaLuján, R. (2017). Propiedades psicométricas del Behavioral Regulation in Exercise Questionnaire-2en universitariosmexicanos. Retos, 34(34), 80-84. doi.org/ 10.47197/ retos. V0i34.55794

Domínguez-Alonso, J., López-Castedo, A., \& PortelaPino, I. (2017).Validación del autoinformedemotivosparalapráctica del ejercicio físico con adolescentes (AM PEF): diferencias por género, edad y ciclo escolar. Retos, 33(33), 273-278. doi.org/ 10.47197/ retos. v0i33.58963

Dominguez-Lara, S.A. (2018). Ítemúnico deansiedad anteexá menes: evidencias devalidez convergenteeincremental en estudiantesuniversitarios. Educación Médica, 19(5), 264-270. Dominguez-Lara, S., N avarro-Loli, J., \& PradaCChapoñan, R. (2019). Ítem único deautoeficaciaacadémica: evidenciasadicionalesdevalidezcon el modelo BigFiveen estudiantesuniversitarios. Avaliaçao Psicologica: Interamerican Journal of Psychological Assessment, 18(2), 210-217.

Dunn,T.J., Baguley,T., \&Brunsden,V.(2014). From alphato ome 
ga: apractical solution to the pervasive problem of internal consistencyestimation. British journal of psychology, 105(3),399412. https/ / doi.org/ 10.1111/ bjop. 12046

Fernández, M. E.,Van Damme, L., Daset, L., \&Vanderplasschen, W. (2020). Predictors of D omain-Spe-cific Aspects of SubjectiveWell-Being among School GoingAdolescentsin U ruguay. AvancesesPsicología Latinoamericana, 38 (1), 85-99.

Fornell, C., \& Larcker, D. F. (1981). Evaluatingstructural equation modelswith unobservablevariablesand measurementerror. Journal of Marketing Research, 18(1), 39-50. doi.org/ 10.2307/ 3151312.

Gallegos, W. (2017). Psicologíaclínicay psicoterapia: Revisión epistemológicay aportesdelapsicologíapositiva. Revistape ruana depsicología y trabajo social, 2(1), 137-153.

García, D. \& Serrano, M. (2017). Lainteligenciaemocional: capa cidad parael desarrollo humano en el marco delaorientación eductiva. Revista Convocación, 30,VII,56-67.

GarcíaÁlvarez, D.\& Soler, M.J. (2020). Programacreciendo fuertes, desarrollo positivo adolescentey educación: un entrama do saludable. Arté. Revista Digital del D octoradoen Educación de la Universidad Central del/enezuela, 6 (12), 163-182.

GarcíaÁlvarez, D., Hernández-Lalinde, J., Espinoza-Castro,J. \& Soler, M. (2020). Salud mental en la adolescencia montevideana: unamiradadesdeel bienestar psicológico. Archivos/enezolanosdeFarmacología yTerapéutica, 39(2), 182-190.

GarcíaÁlvarez, D., Soler, M. J., \& Cobo-Rendón, R. (2020). Promoting character strengths through the Growing Up Strong Program:Thestudents' perspective Revisa deEstudios elnvestigación en Psicología y Educación, 7(1), 84-97.

GarcíaÁlvarez, D., Soler, M., \& Rendón, R. (2019). Bienestar psicológico en adolescentes: relaciones con autoestima, autoeficacia, malestar psicológico y śntomasdepresivos. Re vista deO rientación Educacional, 33(63), 23-43.

Henseler, J., Ringle, C. M ., \& Sarstedt, M. (2015).A new criterion for assessing discriminantvalidity in variance-based structural equation modeling Journal of theAcademy of M arketing Science, 43(1), 115-135. doi.org/ 10.1007/ s11747-014-0403-8.

HernándezSampieri, R., FernándezCollado, C., \& BaptistaLucio, P. (2014). Metodología de la investigación. M cG raw-Hill Education.

Hernández-Lalinde,J. D., Espinosa Castro,J. F., GarcíaÁlvarez, D., Bermúdez-Pirela,V.J. (2019). Sobreel uso adecuado dela regresión linea: conceptualización básicamedianteun ejemplo aplicado a las ciencias de la salud. Archivosvenezolanos de Farmacología yTerapéutica, 38(5), 609-614.

Hernández-Ladinde,J. D., Espinosa-Castro,J. F., Peñaloza Tarazona, M. E., Rodríguez,J., Chacón-Rangel,J. G., Toloza-Sierra, C.A., Arenas-Torrado,M.K.,Carrillo-Sierra, S.M.,Bermúdez-Pirela, V.J. (2018). Sobreel uso adecuado del coeficientedecorrela ción dePearson: definición, propiedadesysuposiciones Archivos/enezolanosdeFarmacología yTerapéutica, 37(5), 587-595.

Hosseinabad, N., N ajafi,M .,\& Rezaa,A.M. (2019).Theeffectiveness of training program of positive youth development on life satisfaction and self-efficacyin adolescents. Journal of Psychological Science, 17(72), 927-936.

Kankainen,A.,Takkinen, S. \& O ja, H. (2004). On Mardia'stests of multinormality. EnM. Hubert, G. Pison, A. Struyf, \&S.Van Aelst(Eds), TheoryandApplicationsof Recent Robus Methods(pp. 153-164). Birkhäuser.

Leffert, N., Benson, P. L., Scales, P. C., Sharma, A. R., Drake, D. R., $\&$ Blyth, D.A. (1998). Developmental assets. M easurement and prediction of risk behaviorsamongadolescents. Applied Developmental Science, 2(4), 209-230.

Lerner,J.V., Phelps, E., Forman,Y., \& Bowers, E. P. (2009). Positive youth development. InR. M. Lerner \& L. Steinberg(Eds.), Handbook of adolescent psychology: Individual bases of adolescent development (p. 524-558). JohnW iley \& Sons Inc. doi.org/ 10.1002/ 9780470479193 . adl psy001016

Li, C. (2013). Little'stest of missing completely at random. The Stata Journal, 13(4), 795-809. doi.org/ 10.1177/ $1536867 \times 1301300407$

Lombas,A. S., Jiménez,T. I.,Arguís-Rey, R., Hernández-Paniello, S., Valdivia_Salas, S., \& M artín-Albo, J. (2019). Impact of the HappyClassroomsProgrammeon PsychologicalWell-being, SchoolAggression, and Classroom Climate Mindfulness 10(8), 1642-1660

Mazloomy, S.S.,Atabay, R.A., Rahimi, M., Fallahzadeh, H., \&Vææzi, A. (2019). The relationship between developmental assets and well-beinginadolescentfemalestudentsinYazd, Iran.journal of IndianAssociation for Child \& Adolescent M ental H ealth, 15(3), 9-32.

Montero, I., \& León, 0. G. (2007).A guidefor namingresearch studiesin Psychology. International Journal of Clinical and $\mathrm{H}$ ealth Phsycology,7 (3), 847-862.

Mord,J. (2019). Revisión deloscriteriosparavalidezconvergente estimada a través de la Varianza Media Extraída. Psychologia, 13(2), 25-41.

O liva, A. (2015). Los activos parala promoción del desarrollo positivo adolescente. Revista M etamorfosis: Revistadel Centro ReinaSofíasobreAdolescenciayJ uventud, (3), 32-47.

O liva,A.,Antolín-Surez, L., \& López,A.. (2012). Development andValidation of aScalefor theM easurement ofAdolescents' DevelopmentalAssetsintheN eighborhood. Social Indicators Research, 106(3), 563-576.

Oliva,A.,AntolínSuárez, L., PertegalVega, M. Á., RíosBermúdez, M., Parraliménez, Á., Hernando Gómez, Á., \& ReinaFlores, M. D. C. (2011a). Instrumentos para la evaluación de la salud mental y el desarrollo positivo adolescente y los activos que 10 promueven. Andalucía: ConsejeríadeSalud.

Oliva,A., Pertegal, M.A., Hernando,A., Reina, M. C.,AntolínSuárez, Parra, A. \& Ríos, M. (2015). Un estudio empírico sobreel desarrollo positivo adolescentey losactivosquelo promueven. En (A. O liva, Coord.). D esarrollo PositivoAdolescente(pp. 119-138). M adrid: Síntesis. 
Oliva,A.,Pertega,M.,.Antolín, L., Reina, M.., Ríos, M., Hernando, Á., Parra, A., Pascual, D. \& EstévezCampos, R. M. (2011b). Desarrollo positivo adolescente y los activos que lo promueven: un estudioen centrosdocentesandaluces. Sevilla: JuntadeAndalucía

Oliva,A., Povedano,A., Suarez, C.,Antolín- Surez, L., RodríguezMeirinhos,A., \& M usito, G. (2020). Bienestary desarrollo positivo adolescentedesdeuna perspectiva degénero. U n estudio cuantitativo. Madrid: Centro ReinaSofíasobreAdolescenciayJuventud, Fad.

Oliva,A., Ríos, M.,Antolín, L., Parra, Á., Hernando, Á., \& Pertegal, M. Á. (2010). Másaládel déficit: Construyendo un modelo dedesarrollo positivo adolescente. InfanciayAprendizaje,33(2), 223-234.

Oliva, A., Suárez, L. A., Díaz, A. P., Relinque, C. S., del Moral Arroyo, G., Rodríguez-M eirinhos, A., ... \& O choa, G. M. (2017). Bienestar y desarrollo positivo adolescentedesdeuna perspectiva degénero: un estudio cual itativo. Centro ReinaSofíasobre AdolescenciayJ uventud, en colaboración, U niversidad de Sevillay Universidad Pablo deO lavide.

O man, R. F.,Vesely, S. K., McLeroy, K.R., HarrisWyatt,V.,Aspy, C.B., Rodine, S., \& Marshall,L. (2002). ReliabilityandValidity of theYouthAsset Survey (YAS). Journal of Adolescent H ealth, 31(3), 247-255.

O rganización PanamericanadelaSalud. (2018). Aceleración mundial delasmedidaspara promover la salud delosadolescentes (Guía AA-HA!):O rientación para la aplicación en lospaísss. Washington, D.C.

Pertegal-Vega, M., Gómez, Á., \& O liva.A. (2011). Desarrolloy validación deunaescaladeevaluación delosactivosy fortalezasescolaresdesdelaperspectivadel profesorado. Apuntesde Psicología, 29(3), 379-395.

Proctor, C.,T sukayama, E.,Wood,A. M., Maltby, J., Eades,J. F., y Linley, P.A. (2015). Gimnasiaparalasfortalezas: impacto de unaintervención basadaen lasfortalezas del carácter sobrela satisfacción con laviday el bienestar delosadolescentes. RET, Revista deToxicomanías, 75, 10-23.

Proctor, C., Tsukayama, E.,Wood,A. M., M attby,J., Eades, J. F., y Linley, P.A. (2011). Strengthsgym:Theimpact of acharacter strengths-based intervention on the lifesatisfaction and wellbeing of adolescents. The Journal of PositivePsychology,6 (5), 377388.

Reina, M., Oliva, A., \& Parra, Á. (2010). Percepciones de autoevaluación:Autoestima, autoeficaciay satiffacción vital en laadolescencia. Psychology, Society, E Education,2(1), 55-69.

Reininger, B., Evans, A.E., Griffin, S. F.,Valois, R. F.,Vincent, M. L., ParraMedina, D., ...\&Zullig, K.). (2003). Developmentofa YouthSurvey to MeasureRisk Behaviors,AttitudesandAssets. Examining Multiple Influences. Health Education Research, 18(4), 461-476.

Ruiz-Aranda, D., Cabello, R., Salguero, J.M., Castillo, R., Extremera, N. \& Fernández-Berroca, P. (2010). Losadolescentesmalagueñosantelasdrogas la influencia dela inteligencia emo-
cional.M adrid:TEA ediciones

Ryff,C. D. (2014). Psychological well-beingrevisited:Advancesin the science and practice of eudaimonia. Psychotherapy and Psychosomatics, 83(1), 10-28.

Sánchez, C. (2014). Revisónteóricasobreel estudio delasforta lezashumanasen diferentesetapasevolutivas. Trabajo Fin de Grado,U niversidaddejaén.

Sánchez, 0.\& Méndez, F. (2009). 0 ptimismasaProtectiveFactor inChild andAdolescentD epression. Clínica y Salud, 20(3), 273-280.

Satorra, A. \& Bentler, P. M. (2001). A scaled differencechi-square teststatisticfor momentstructureanalysis. Psychomerika, 66(4), 507-514. doi.org/ 10.1007/ BF02296192.

Scales, P.C. (2011).Youthdevelopmental assetsin doba perspective: Resultsfrom international adaptations of the Developmenta AssetsProfile. Child IndicatorsResearch, 4(4), 619-645

Scales, P. C., Benson, P. L., Leffert, N., \& Blyth, D. A. (2000). Contribution of developmental assets to the prediction of thriving amongadolescents. Applied Developmental Science, 4(1), 27-46.

Scheier, M. F., Carver, C. S., \& Bridges, M.W. (1994). Distinguishing optimism from neuroticism (and trait anxiety, self-mastery, and self-esteem): a reevaluation of the Life 0 rientation Test. Journal of personality and social psychology, 67 (6), 10631078.

SearchInstitute. (2005). D evelopmental assetsprofile U ser manual. Minneapolis, MN: Search Institute.

Seligman, M. E., Ernst, R. M., Gillham, J., Reivich, K., \& Linkins, M. (2009). Positive education: Positive psychology and classroom interventions. Oxford Review of Education, 35(3), 293-311.

Soares,A. S., Pais-Ribeiro, J. L., \& Silva, I. (2019). Developmental assetspredictors of lifesatisfaction in adolescents. Frontiersin Psychology, 10, 236-236.

Trigueros, R.,Aguilar-Parra,J., González-Santos,.., \& Cangas,A. (2019).Validación yadaptación delaescaladecontrol psicológico del profesor hacialasclasesdeeducación físicay su efecto sobrelasfrustracionesdelasnecesidadespsicológicasbásicas. Retos, 37(37), 167-173. doi.org/ 10.47197/ retos. v37i37.71550

Wanous, J. P., \& Hudy, M. J. (2001). Single-Item Reliability:A ReplicationandExtension. Organizational Research M ethods4(4), 361-375. https:/ / doi.org/ 10.1177/ 109442810144003

Waters, E., \& Sroufe, L. A. (1983). Social competence as a developmental construct. Developmental Review, 3(1), 79-97.

W hite, M.A., \&W aters, L. E. (2015). A casestudy of'TheGood School:'Examples of the use of Peterson's strengths-based approach with students Thejournal of PositivePsychology,10(1), 69-76. 